\title{
Discrete Transparent Boundary Conditions for General Schrödinger-type Equations
}

\author{
MATTHIAS EHRHARDT* \\ Fachbereich Mathematik, TU Berlin, MA 6-2, Straße des 17. Juni 136, D-10623 Berlin, Germany
}

(Received 13 August 1997; In final form 1 December 1998)

\begin{abstract}
Transparent boundary conditions (TBCs) for general Schrödinger-type equations on a bounded domain can be derived explicitly under the assumption that the given potential $V$ is constant on the exterior of that domain. In 1D these boundary conditions are nonlocal in time (of memory type).

Existing discretizations of these TBCs have accuracy problems and render the overall Crank - Nicolson finite difference method only conditionally stable. In this paper a novel discrete TBC is derived directly from the discrete whole-space problem that yields an unconditionally stable scheme. Numerical examples illustrate the superiority of the discrete TBC over other existing consistent discretizations of the differential TBCs.

As an application of these boundary conditions to wave propagation problems in underwater acoustics results for the so-called standard and wide angle "parabolic" equation (SPE, WAPE) models are presented.
\end{abstract}

Keywords: Schrödinger equation, transparent boundary conditions, finite differences, discrete transparent boundary conditions, underwater acoustics, wide angle "parabolic" equation

\section{INTRODUCTION}

This paper is concerned with the construction and discretization of transparent boundary conditions (TBCs) for general Schödinger-type pseudodifferential equations in $1 \mathrm{D}$ of the form

$$
\begin{aligned}
-i \psi_{t}= & \left(\frac{p_{0}+p_{1}(\Delta-V(x, t))}{1+q_{1}(\Delta-V(x, t))}-1\right) \psi, \\
& x \in \mathbb{R}, t>0, \\
\psi(x, 0)= & \psi^{I}(x),
\end{aligned}
$$

\footnotetext{
*e-mail: ehrhardt@,math.tu-berlin.de
}

where the real coefficients $p_{0}, p_{1}, q_{1}$ are constant and the complex valued "potential" $V$ is assumed to be given. As a special case $\left(q_{1}=0\right)$ the Schrödinger equation of quantum mechanics is included. In quantum mechanics the transparent boundary conditions for the Schrödinger equation could be applied for the coupling of two electron waveguides [26].

Equations of the form (1.1) arise from "parabolic" equation (PE) models which have been widely used for wave propagation problems in 
various application areas, e.g., seismology [8, 9], optics and plasma physics ( $c f$. the references in [6]). In underwater acoustics they appear as wide angle approximation to the Helmholtz equation in cylindrical coordinates and are called wide angle parabolic equations (WAPE) [16]. There, the radial variable $r$ plays the role of the time in (1.1), and the axial variable $z$ the role of the spatial variable $x$.

If one wants to solve numerically a whole-space evolution problem, one has to restrict the computational domain by introducing artificial boundary conditions. If the initial data is supported on this finite domain $\Omega$, one can construct BCs on $\partial \Omega$ with the objective to thereby approximate the exact solution of the whole-space problem, restricted to $\Omega$. Such BCs are called absorbing boundary conditions (ABCs). If the approximate solution coincides on $\Omega$ with the exact solution, one refers to these BCs as transparent boundary conditions (TBCs). The TBC for the Schrödinger equation was independently derived by several authors from various application fields $[4,7,15,17,19,21]$.

The main motivation of this paper is the numerical discretization of transparent boundary conditions. While TBCs fully solve the problem of cutting off the $x$-domain for the analytical equation, their numerical discretization is far from trivial. There are two problems of these existing consistent discretizations of the continuous TBC. First, the discretized TBC for the Schrödinger equation destroys the unconditional stability of the underlying Crank-Nicolson scheme [17]. Secondly, these available discretizations suffer from reduced accuracy (in comparison to the discretized whole-space problem) and induce numerical reflections at the boundary, particularly when using coarse grids.

In this paper we present a new approach which overcomes both the stability problem and reduced accuracy. We construct discrete transparent boundary conditions (DTBC) for a Crank-Nicolson finite difference discretization of (1.1) such that the overall scheme is unconditionally stable and as accurate as the discretized whole-space problem. The resulting DTBC is a generalization of the
DTBC for the Schrödinger equation in [4]. The same strategy was used in [12] for advection diffusion equations and in [11] for the wave equation in the frequency domain.

The paper is organized as follows: $\operatorname{In} \S 2$ we review the TBC for the $1 \mathrm{D}$ Schrödinger equation. In $\S 3$ the discrete TBCs are derived and analyzed; their application to underwater acoustics is shown in $\S 4$. Various numerical examples in $\S 3$ illustrates the superiority of our DTBC over existing discretizations.

\section{TRANSPARENT BOUNDARY CONDITIONS}

In this Section we shall sketch the derivation of the TBC. For simplicity we will only treat the case of the Schrödinger equation

$$
\begin{aligned}
i \hbar \psi_{t} & =-\frac{\hbar^{2}}{2} \Delta \psi+V(x, t) \psi, \quad x \in \mathbb{R}, \quad t>0, \\
\psi(x, 0) & =\psi^{I}(x) .
\end{aligned}
$$

We make the two basic assumptions that the initial data $\psi^{I}$ is supported in $0<x<L$ and that the given electrostatic potential is constant outside this finite domain: $V(x, t)=0$ for $x \leq 0, V(x, t)=V_{L}$ for $x \geq L$.

The first step is to cut the original whole-space problem (1.1) into three subproblems, the interior problem on $\Omega$, and a left and right exterior problem. They are coupled by the assumption that $\psi, \psi_{x}$ are continuous across the artificial boundary. The interior problem reads

$$
\begin{aligned}
i \hbar \psi_{t}= & -\frac{\hbar^{2}}{2} \Delta \psi+V(x, t) \psi, \\
& 0<x<L, \quad t>0, \\
\psi(x, 0)= & \psi^{I}(x) \\
\psi_{x}(0, t)= & \left(T_{-} \psi\right)(0, t), \\
\psi_{x}(L, t)= & \left(T_{+} \psi\right)(L, t) .
\end{aligned}
$$


$T_{ \pm}$denote the Dirichlet-to-Neumann maps at the boundaries, and they are obtained by solving the two exterior problems:

$$
\begin{gathered}
i \hbar v_{t}=-\frac{\hbar^{2}}{2} \Delta v+V_{L} v, \\
x>L, t>0, \\
v(x, 0)=0, \\
v(L, t)=\Phi(t), \\
t>0, \Phi(0)=0, \\
v(\infty, t)=0, \\
\left(T_{+} \Phi\right)(t)=v_{x}(L, t),
\end{gathered}
$$

and analogously for $T_{-}$. Since the potential is constant in the exterior problems, we can solve them explicitly by the Laplace method and thus obtain the two boundary operators $T_{ \pm}$needed in (2.2).

The Laplace transformation of $v$ is given by

$$
\hat{v}(x, s)=\int_{0}^{\infty} v(x, t) \mathrm{e}^{-s t} d t
$$

where we set $s=\eta+i \xi, \xi \in \mathbb{R}$ and $\eta>0$ is fixed, with the idea to later perform the limit $\eta \rightarrow 0$. Now the right exterior problem (2.3) is transformed to

$$
\begin{gathered}
\hat{v}_{x x}+i \frac{2}{\hbar}\left(s+i \frac{V_{L}}{\hbar}\right) \hat{v}=0, \quad x>L, \\
\hat{v}(L, s)=\hat{\Phi}(s) .
\end{gathered}
$$

Since its solutions have to decrease as $x \rightarrow \infty$, we obtain

$$
\hat{v}(x, s)=\mathrm{e}^{-\sqrt[+]{-i \frac{2}{\hbar}\left(s+i \frac{V_{L}}{\hbar}\right)(x-L)}} \hat{\Phi}(s) .
$$

Hence the Laplace-transformed Dirichlet-to-Neumann operator $T_{+}$reads

$$
\begin{aligned}
\widehat{T_{+} \Phi}(s) & =\hat{v}_{x}(L, s) \\
& =-\sqrt{\frac{2}{\hbar}} \mathrm{e}^{-i \pi / 4} \sqrt[+]{s+i \frac{V_{L}}{\hbar}} \hat{\Phi}(s),
\end{aligned}
$$

and $T_{-}$is calculated analogously. Here, $\sqrt[+]{ }$ denotes the branch of the square root with nonnegative real part.
An inverse Laplace transformation yields the right TBC at $x=L$ :

$$
\begin{aligned}
\psi_{x}(L, t)= & -\sqrt{\frac{2}{\hbar \pi}} \mathrm{e}^{-i \pi / 4} \mathrm{e}^{-i\left(V_{L} / \hbar\right) t} \\
& \frac{d}{d t} \int_{0}^{t} \frac{\psi(L, \tau) \mathrm{e}^{i\left(V_{L} / \hbar\right) \tau}}{\sqrt{t-\tau}} d \tau .
\end{aligned}
$$

Similarly, the left TBC at $x=0$ is obtained as

$$
\psi_{x}(0, t)=\sqrt{\frac{2}{\hbar \pi}} \mathrm{e}^{-i \pi / 4} \frac{d}{d t} \int_{0}^{t} \frac{\psi(0, \tau)}{\sqrt{t-\tau}} d \tau .
$$

These BCs are non-local in $t$ and of memory-type, thus requiring the storage of all previous time levels at the boundary in a numerical discretization.

We remark that (2.8) is equivalent to the impedance boundary condition [19]:

$$
\psi(L, t)=-\sqrt{\frac{\hbar}{2 \pi}} \mathrm{e}^{i \pi / 4} \int_{0}^{t} \frac{\psi_{x}(L, t-\tau) \mathrm{e}^{-i\left(V_{L} / \hbar\right) \tau}}{\sqrt{\tau}} d \tau .
$$

It should be noted that the potential-free Schrödinger equation can formally be factorized into left and right travelling waves (cp. [7]):

$$
\left(\frac{\partial}{\partial x}-\sqrt{\frac{2}{\hbar}} \mathrm{e}^{-(\pi / 4) i} \sqrt{\frac{\partial}{\partial t}}\right)\left(\frac{\partial}{\partial x}+\sqrt{\frac{2}{\hbar}} \mathrm{e}^{-(\pi / 4) i} \sqrt{\frac{\partial}{\partial t}}\right) \psi=0,
$$

where the term (cp. (2.9))

$$
\frac{1}{\sqrt{\pi}} \frac{d}{d t} \int_{0}^{t} \frac{\psi(\tau)}{\sqrt{t-\tau}} d \tau=: \sqrt{\frac{d}{d t}} \psi
$$

can be interpreted as a fractional (1/2) time derivative.

The existence of a solution to the 1D Schrödinger equation with the TBCs (2.8), (2.9) is clear from the used construction. For regular enough initial data, e.g., $\psi^{I} \in H^{1}(0, L)$, the whole-space solution $\psi(x, t)$ will satisfy the TBCs at least in a weak sense. The uniqueness of the solution is, however, not trivial. In order to prove uniform 
boundedness of $\|\psi(., t)\|_{L^{2}(0, L)}$ in $t$ we will need the following simple lemma which states that the kernel_of the Dirichlet-to-Neumann operator $\mathrm{e}^{i \pi / 4} \sqrt{\frac{d}{d t}}$ is of positive type in the sense of memory equations (see, e.g. [14]).

Lemma 1 ([4]) For any $T>0$, let $u \in H^{(1 / 4)}(0, T)$ with the extension $u(t)=0$ for $t>T$. Then

$$
\operatorname{Re}\left\{\mathrm{e}^{i \pi / 4} \int_{0}^{\infty} \bar{u}(t) \frac{d}{d t}\left[\int_{0}^{t} \frac{u(s)}{\sqrt{t-s}} d s\right] d t\right\} \geq 0 .
$$

A straight forward calculation with the Schrödinger equation now shows

$$
\|\psi(t)\|_{L^{2}(0, L)} \leq\left\|\psi^{I}\right\|_{L^{2}(0, L)}, \quad t>0
$$

and this implies uniqueness of the solution to the Schrödinger IBVP. (2.14) reflects the fact that some of the initial mass or particle density $n(x, t)=|\psi(x, t)|^{2}$ leaves the computational domain $[0, L]$ during the evolution. In the wholespace problem, $x \in \mathbb{R},\|\psi(t)\|_{L^{2}(\mathbb{R})}$ is of course conserved.

\section{DISCRETE TRANSPARENT BOUNDARY CONDITIONS}

In this Section we shall discuss how to discretize the TBC (2.8) in conjunction with a CrankNicolson scheme. For simplicity only, we restrict ourselves to the case of the Schrödinger equation (2.1) and review the derivation of the DTBC from [4].

With the uniform grid points $x_{j}=j \Delta x, t_{n}=n \Delta t$, and the approximations $\psi_{j}^{n} \sim \psi\left(x_{j}, t_{n}\right)$ the discretized Schrödinger equation (2.1) reads:

$$
\begin{aligned}
-i R\left(\psi_{j}^{n+1}-\psi_{j}^{n}\right)= & \Delta^{2} \psi_{j}^{n+1}+\Delta^{2} \psi_{j}^{n} \\
& +w V_{j}^{n+1 / 2}\left(\psi_{j}^{n+1}+\psi_{j}^{n}\right),
\end{aligned}
$$

with

$$
\begin{gathered}
R=\frac{4 \Delta x^{2}}{\hbar} \frac{\Delta t}{\Delta t}, \quad w=-\frac{2 \Delta x^{2}}{\hbar^{2}}, \\
V_{j}^{n+1 / 2}=V\left(x_{j}, t_{n+1 / 2}\right),
\end{gathered}
$$

where $\Delta^{2} \psi_{j}^{n}=\psi_{j+1}^{n}-2 \psi_{j}^{n}+\psi_{j-1}^{n}$, and $R$ is proportional to the parabolic mesh ratio. For our analysis, one of the main advantages of this second order (in $\Delta x$ and $\Delta t$ ) scheme is, that it is unconditionally stable, and an easy calculation shows that it preserves the discrete $L^{2}$-norm: $\left\|\psi^{n}\right\|_{2}^{2}=$ $\Delta x \sum_{j \in \mathbb{Z}}\left|\psi_{j}^{n}\right|^{2}$, which is the discrete analogue of the mass conservation property of (2.1).

We remark that most existing discretization schemes for the Schrödinger equation with TBCs are also based on the Crank-Nicolson finite differences $([7,17,20])$.

We shall now compare three strategies to discretize the TBC (2.8) with its mildly singular convolution kernel. First we review a known discretization from the literature, where the analytic $\mathrm{TBC}$ in the equivalent form (2.10) at $L=J \Delta x$ was discretized in an ad-hoc fashion. In [17] Mayfield proposed the approximation

$$
\begin{aligned}
& \int_{0}^{t_{n}} \frac{\psi_{x}\left(L, t_{n}-\tau\right) \mathrm{e}^{-i\left(V_{L} / \hbar\right) \tau}}{\sqrt{\tau}} d \tau \\
& \quad \approx \frac{1}{\Delta x} \sum_{m=0}^{n-1}\left(\psi_{J}^{n-m}-\psi_{J-1}^{n-m}\right) \mathrm{e}^{-i\left(V_{L} / \hbar\right) m \Delta t} \int_{t_{m}}^{t_{m+1}} \frac{d \tau}{\sqrt{\tau}},
\end{aligned}
$$

which leads to the following discretized $T B C$ for the Schrödinger equation:

$$
\psi_{J}^{n}-\psi_{J-1}^{n}=\frac{\Delta x}{2 B \sqrt{\Delta t}} \psi_{J}^{n}-\sum_{m=1}^{n-1}\left(\psi_{J}^{n-m}-\psi_{J-1}^{n-m}\right) \ell_{m}
$$

with

$$
B=-\sqrt{\frac{\hbar}{2 \pi}} \mathrm{e}^{i \pi / 4}, \quad \ell_{m}=\frac{\mathrm{e}^{-i\left(V_{L} / \hbar\right) m \Delta t}}{\sqrt{m+1}+\sqrt{m}} .
$$


On the fully discrete level this BC is not perfectly transparent any more. For the resulting scheme Mayfield obtained the following result:

Theorem 2 ([17]) The discretization scheme (3.1), (3.3) is stable, if and only if

$$
4 \pi \hbar \frac{\Delta t}{\Delta x^{2}} \in \bigcup_{j \in \mathbf{N}_{0}}\left[(2 j+1)^{-2},(2 j)^{-2}\right] .
$$

This shows that the chosen discretization of the TBC destroys the unconditional stability of the underlying Crank-Nicolson scheme and one can expect a similar behaviour for (1.1).

In the semi-discrete approach of Schmidt and Deuflhard [22] a TBC is derived for the semidiscretized (in $t$ ) Schrödinger equation. This method also applies for a nonuniform (e.g., adaptive) interior scheme and it admits a time-dependent potential in the exterior domain (i.e., $V_{L}=V_{L}(t)$ ). While being unconditionally stable (in conjunction with an interior finite element scheme) [23], it still exhibits small residual reflections at the artificial boundary.

Instead of using an ad-hoc discretization of the analytic TBC like (3.2) we will construct discrete $T B C s$ of the fully discretized whole-space problem. Our new strategy solves both problems of the discretized $T B C$ at no additional computational costs. With our DTBC the numerical solution on the computational domain $0 \leq j \leq J$ exactly equals the discrete whole-space solution (on $j \in \mathbb{Z}$ ) restricted to the computational domain $0 \leq j \leq J$. Therefore, our overall scheme avoids any numerical reflections at the boundary and inherits the unconditional stability of the whole-space CrankNicolson scheme (see Theorem 4).

Consequently, when considering the discretization of TBCs, it should be a standard strategy to derive the discrete $T B C S$ of the fully discretized problem, rather then attempting to discretize the differential TBC. A comparison of these two strategies for a $1 \mathrm{D}$ wave propagation problem is given in [11].

To derive the discrete TBC we will now mimick the derivation of the analytic TBC from Section 2 on a discrete level. Again, we will only consider the right BC. In analogy to the continuous problem we assume for the potential and initial data: $V_{j}^{n}=$ $V_{L}=$ const, $\psi_{j}^{0}=0, j \geq J-1$, and solve the discrete right exterior problem by using the $Z$-transform:

$$
\mathcal{Z}\left\{\psi_{j}^{n}\right\}=\hat{\psi}_{j}(z):=\sum_{n=0}^{\infty} \psi_{j}^{n} z^{-n}, \quad z \in \mathbb{C},|z|>1
$$

Hence, the $Z$-transformed finite difference scheme (3.1) for $j \geq J$ reads

$$
\begin{aligned}
& (z+1) \Delta^{2} \hat{\psi}_{j}(z)=-i R[z-1+i \kappa(z+1)] \hat{\psi}_{j}(z), \\
& \kappa=\frac{\Delta t}{2} \frac{V_{L}}{\hbar}
\end{aligned}
$$

The two linearly independent solutions of the resulting second order difference equation take the form $\hat{\psi}_{j}(z)=\nu_{1,2}^{j}(z), j \geq J$, where $\nu_{1,2}(z)$ solve

$$
\nu^{2}-2\left[1-\frac{i R}{2}\left(\frac{z-1}{z+1}+i \kappa\right)\right] \nu+1=0 .
$$

For the decreasing mode (as $j \rightarrow \infty$ ) we have to require $\left|\nu_{1}(z)\right|<1$ and obtain the $Z$-transformed DTBC as

$$
\hat{\psi}_{J-1}(z)=\nu_{1}^{-1}(z) \hat{\psi}_{J}(z)
$$

In a tedious calculation (3.8) can be inverse transformed explicitly:

TheOREM 3 ([4]) The left $(a t j=0)$ and right (at $j=J$ ) discrete TBCs for the Crank-Nicolson discretization (3.1) of the $1 D$ Schrödinger equation are

$$
\begin{aligned}
& \psi_{1}^{n}=\sum_{k=1}^{n} \psi_{0}^{k} \ell_{0}^{n-k}-\psi_{1}^{n-1}, \\
& \psi_{J-1}^{n}=\sum_{k=1}^{n} \psi_{J}^{k} \ell_{J}^{n-k}-\psi_{J-1}^{n-1}, \quad n \geq 1,
\end{aligned}
$$


with

$$
\begin{aligned}
& \ell_{j}^{n}= {\left[1-i \frac{R}{2}+\frac{\sigma}{2}\right] \delta_{n}^{0}+\left[1+i \frac{R}{2}+\frac{\sigma}{2}\right] \delta_{n}^{1} } \\
&+\alpha_{j} \mathrm{e}^{-i n \varphi_{j}} \frac{P_{n}\left(\mu_{j}\right)-P_{n-2}\left(\mu_{j}\right)}{2 n-1}, \\
& \varphi_{j}= \arg \frac{2 R\left(\sigma_{j}+2\right)}{R^{2}-4 \sigma_{j}-\sigma_{j}^{2}}, \\
& \mu_{j}=\frac{R^{2}+4 \sigma_{j}+\sigma_{j}^{2}}{\sqrt{\left(R^{2}+\sigma_{j}^{2}\right)\left(R^{2}+\left[\sigma_{j}+4\right]^{2}\right)}} \\
& \sigma_{j}=\frac{2 \Delta x^{2}}{\hbar^{2}} V_{j}, \\
& \alpha_{j}=\frac{i}{2} \sqrt[4]{\left(R^{2}+\sigma_{j}^{2}\right)\left(R^{2}+\left[\sigma_{j}+4\right]^{2}\right)} \mathrm{e}^{i \varphi_{j} / 2}, \\
& j=0, J .
\end{aligned}
$$

$P_{n}$ denotes the Legendre polynomials $\left(P_{-1} \equiv P_{-2} \equiv\right.$ $0)$ and $\delta_{n}^{j}$ the Kronecker symbol.

The $P_{n}$ only have to be evaluated at the two values $\mu_{0}, \mu_{\mathrm{J}}$, and hence the numerically stable recursion formula for the Legendre polynomials can be used. Using asymptotic properties of the Legendre polynomials one finds $\ell_{0}^{n}, \ell_{J}^{n}=O\left(n^{-3 / 2}\right)$, which agrees with the decay of the convolution kernel in the differential TBCs (2.8), (2.9) (after an integration by parts).

Since the discrete whole-space solution satisfies the discrete TBCs (3.9), it is trivial that the implicit schemes (3.1), (3.9) for the IBVP can be solved at each time level $n$. To prove unique solvability and stability of the scheme, one can derive a discrete analogue of (2.14). We then have the main result of this Section:

Theorem 4 ([4]) The solution of the discretized Schrödinger equation (3.1) with the discrete TBCs (3.9) is uniformly bounded

$$
\left\|\psi^{n}\right\|_{2}^{2}:=\Delta x \sum_{j=1}^{J-1}\left|\psi_{j}^{n}\right|^{2} \leq\left\|\psi^{0}\right\|_{2}^{2}, \quad n \geq 1
$$

and the scheme is thus unconditionally stable.

\section{APPLICATION TO UNDERWATER ACOUSTICS}

In oceanography one wants to calculate the underwater acoustic pressure $p(z, r)$ emerging from a time-harmonic point source located in the water at $\left(z_{s}, 0\right)$. Here, $r>0$ denotes the radial range variable and $0<z<z_{b}$ the depth variable. The water surface is at $z=0$, and the (horizontal) sea bottom at $z=z_{b}$. We denote the local sound speed by $c(z, r)$, the density by $\rho(z, r)$, and the attenuation by $\alpha(z, r) \geq 0 . n(z, r)=c_{0} / c(z, r)$ is the refractive index, with a reference sound speed $c_{0}$ (usually the smallest sound speed in the model). Then the reference wave number is $k_{0}=2 \pi f / c_{0}$, where $f$ denotes the (usually low) frequency of the emitted sound.

The pressure satisfies the Helmholtz equation

$$
\begin{aligned}
\frac{1}{r} \frac{\partial}{\partial r}\left(r \frac{\partial p}{\partial r}\right) & +\rho \frac{\partial}{\partial z}\left(\rho^{-1} \frac{\partial p}{\partial z}\right) \\
+k_{0}^{2} N^{2} p & =0, \quad r>0
\end{aligned}
$$

with the complex refractive index

$$
N(z, r)=n(z, r)+i \alpha(z, r) / k_{0} .
$$

In the far field approximation $\left(k_{0} r \gg 1\right)$ (complex valued) outgoing acoustic field

$$
\psi(z, r)=\sqrt{k_{0} r} p(z, r) \mathrm{e}^{-i k_{0} r}
$$

satisfies the one-way Helmholtz equation:

$$
\psi_{r}=i k_{0}(\sqrt{1-L}-1) \psi, \quad r>0 .
$$

Here, $\sqrt{1-L}$ is a pseudo-differential operator, and $L$ the Schrödinger operator

$$
L=-k_{0}^{-2} \rho \partial_{z}\left(\rho^{-1} \partial_{z}\right)+V(z, r)
$$

with the complex valued "potential" $V(z, r)=$ $1-N^{2}(z, r)$. The evolution equation (4.4) is much easier to solve numerically than the elliptic Helmholtz equation (4.1).

"Parabolic" approximations of (4.4) consist in formally approximating the pseudo-differential 
operator $\sqrt{1-L}$ by rational functions of $L$, which yields a PDE that is easier to discretize than the pseudo-differential equation (4.4), $c f$. $[13,16,24]$. The linear approximation of $\sqrt{1-\lambda}$ by $1-(\lambda / 2)$ gives the narrow angle or standard "parabolic" equation (SPE) of Tappert [24]

$$
\psi_{r}=-\frac{i k_{0}}{2} L \psi, \quad r>0
$$

This Schrödinger equation is a reasonable description of waves with a propagation direction within about $15^{\circ}$ of the horizontal. Rational approximations of the form

$$
(1-\lambda)^{1 / 2} \approx f(\lambda)=\frac{p_{0}-p_{1} \lambda}{1-q_{1} \lambda}
$$

with real $p_{0}, p_{1}, q_{1}$ yield the wide angle "parabolic" equations (WAPE)

$$
\psi_{r}=i k_{0}\left(\frac{p_{0}-p_{1} L}{1-q_{1} L}-1\right) \psi, \quad r>0 .
$$

In the sequel we will repeatedly require the condition $f^{\prime}(0)=p_{0} q_{1}-p_{1}<0$. With the choice $p_{0}=1, p_{1}=(3 / 4), q_{1}=(1 / 4) \quad[(1,1)$-Padé approximant of $\left.(1-\lambda)^{(1 / 2)}\right]$ one obtains the WAPE of Claerbout [8].

Here, the physical problem is posed on the unbounded $z$-interval $(0, \infty)$ and one wishes to restrict the computational domain in the $z$-direction by introducing an artificial boundary at the water-bottom interface $\left(z=z_{b}\right)$, where the wave propagation in water has to be coupled to the wave propagation in the the bottom. At the water surface one usually employs a Dirichlet ("pressure release") BC: $\psi(0, r)=0$.

We shall first address the well-posedness of the evolution problem for the WAPE in the critical non-dissipative case, i.e., for $\alpha=0$ :

$$
\psi_{r}=i k_{0}[f(L)-1] \psi, \quad z>0, \quad r>0,
$$

subject to the surface $\mathrm{BC} \psi(0, r)=0$, and with the rational function $f$ given in (4.7). For simplicity of the analysis we only consider the range-independent situation.
TheOREM 5 ([5]) Assume that the refractive index $n(z)$, the density $\rho(z)>0$, and $\rho^{-1}(z)$ are bounded for $z>0$. Then, the WAPE has a unique solution for all initial data in the weighted $L^{2}$-space $L^{2}\left(\mathbb{R}^{+} ; \rho^{-1} d z\right)$ if and only if the pole of $f(\lambda)$ at $\tilde{\lambda}=q_{1}^{-1}$ is not an eigenvalue of the operator $L$ with Dirichlet BCs at $z=0$.

In applications of underwater acoustics the sound speed $c(z)$ is typically larger in the sea bottom than in the water. Therefore $V(z)$ forms a "potential well" in the water region $0<z<z_{b}$, which typically gives rise to bound states of $L$ that represent the propagating modes of (4.4) and (4.8). All of the corresponding eigenvalues satisfy $0<\lambda_{j}<V_{b}=1-c_{0}^{2} / c_{b}^{2}<1$, if $c_{0}=\min _{z>0} c(z)$. As $q_{1}$ is much smaller than 1 in all practical simulations $(1 / 4$ in the WAPE of Calerbout; also cf. [13]). $\tilde{\lambda}$ usually lies in $\left[V_{b}, \infty\right)$, the continuous spectrum of $L$. Theorem 5 then guarantees the unique solvability of the evolution equation (4.9) for any initial data.

Since the density is typically discontinuous at the water-bottom interface $\left(z=z_{b}\right)$, one requires continuity of the pressure and the normal particle velocity:

$$
\begin{array}{r}
\psi\left(z_{b}-, r\right)=\psi\left(z_{b}+, r\right), \\
\frac{\psi_{z}\left(z_{b}-, r\right)}{\rho_{w}}=\frac{\psi_{z}\left(z_{b}+, r\right)}{\rho_{b}}
\end{array}
$$

where $\rho_{w}=\rho\left(z_{b}-, r\right)$ is the water density just above the bottom and $\rho_{b}$ denotes the constant density of the bottom.

With these matching conditions (4.10) one can easily derive an estimate for the $L^{2}$-decay of solutions to the WAPE, $z>0$. We assume $\rho=\rho(z)$ and apply the operator $1-q_{1} L$ to (4.8):

$$
\begin{aligned}
& {\left[1-q_{1} V+q_{1} k_{0}^{-2} \rho \partial_{z}\left(\rho^{-1} \partial_{z}\right)\right] \psi_{r}} \\
& =i k_{0}\left[p_{0}-1-\left(p_{1}-q_{1}\right) V\right. \\
& \left.\quad+\left(p_{1}-q_{1}\right) k_{0}^{-2} \rho \partial_{z}\left(\rho^{-1} \partial_{z}\right)\right] \psi
\end{aligned}
$$


A simple calculation gives

$$
\begin{gathered}
\partial_{r}\|\psi(., r)\|^{2}=-2 C_{1} \int_{0}^{\infty} \alpha \frac{c_{0}}{c}\left|\tilde{\partial}_{r} \psi\right|^{2} \rho^{-1} d z, \\
C_{1}=\frac{2\left(p_{1}-q_{1}\right)^{2}}{p_{1}-p_{0} q_{1}}
\end{gathered}
$$

for the weighted $L^{2}$-norm ("acoustic energy")

$$
\|\psi(., r)\|^{2}=\int_{0}^{\infty}|\psi(z, r)|^{2} \rho^{-1}(z) d z
$$

i.e., in the dissipation-free case $(\alpha \equiv 0)\|\psi(., r)\|$ is conserved and for $\alpha>0$ and $p_{0} q_{1}-p_{1}<0$ it decays.

Analogously to $\S 2$, we assume that the initial data $\psi^{1}$, which models a point source located at $\left(z_{s}, 0\right)$, is supported in $0<z<z_{b}$. Also, let the bottom region be homogenous, i.e., all physical parameters $c_{b}, \rho_{b}$, and $\alpha_{b}$ be constant for $z>z_{b}$.

Since the SPE is a Schrödinger equation we will only consider the WAPE. In order to derive the TBC for the WAPE we consider (4.11) in the bottom region:

$$
\begin{gathered}
\left(\delta_{b}+q_{1} k_{0}^{-2} \partial_{z}^{2}\right) \psi_{r}=i\left[v_{b}+\left(p_{1}-q_{1}\right) k_{0}^{-1} \partial_{z}^{2}\right] \psi, \\
z>z_{b},
\end{gathered}
$$

with

$$
\begin{aligned}
& \delta_{b}=1-q_{1}\left(1-N_{b}^{2}\right), \\
& v_{b}=k_{0}\left[p_{0}-1-\left(p_{1}-q_{1}\right)\left(1-N_{b}^{2}\right)\right] .
\end{aligned}
$$

The TBC at the bottom for the WAPE reads:

$$
\begin{gathered}
\psi\left(z_{b}, r\right)=-i \eta \frac{\rho_{b}}{\rho_{w}} \psi_{z}\left(z_{b}, r\right) \\
+\beta \eta \frac{\rho_{b}}{\rho_{w}} \int_{0}^{r} \psi_{z}\left(z_{b}, r-\tau\right) \mathrm{e}^{i \theta \tau} \mathrm{e}^{i \beta \tau} \\
\eta=\frac{1}{k_{0}} \sqrt[+]{\frac{q_{1}}{\delta_{b}}}, \quad \beta=-\frac{p_{1}-p_{0} q_{1}}{2 q_{1}} \frac{k_{0}}{\delta_{b}}, \\
\theta=\frac{p_{1}-q_{1}}{q_{1}} k_{0},
\end{gathered}
$$

where $J_{0}, J_{1}$ denote the Bessel functions of order 0 and 1 , respectively. This is a slight generalization of the TBC derived in [21] where $p_{0}$ was equal to 1 . Equivalently, (4.15) can be written as

$$
\begin{aligned}
\psi_{z}\left(z_{b}, r\right)= & i \eta^{-1} \frac{\rho_{w}}{\rho_{b}} \psi\left(z_{b}, r\right) \\
& +\beta \eta^{-1} \frac{\rho_{w}}{\rho_{b}} \int_{0}^{r} \psi\left(z_{b}, r-\tau\right) \mathrm{e}^{i \theta \tau} \mathrm{e}^{i \beta \tau} \\
& \cdot\left[J_{0}(\beta \tau)-i J_{1}(\beta \tau)\right] d \tau
\end{aligned}
$$

Using the asymptotic behaviour of the Bessel functions [1] one finds that the convolution kernel in the TBC (4.16) is $O\left(r^{-3 / 2}\right)$.

Introducing the uniform grid points $z_{j}=j \Delta z$, $r_{n}=n \Delta r$, a Crank-Nicolson discretization of the WAPE (4.11) reads:

$$
\begin{aligned}
& {\left[1-q_{1} V_{j}^{n+1 / 2}+q_{1} k_{0}^{-2} \rho_{j} D_{z}^{0}\left(\rho_{j}^{-1} D_{z}^{0}\right)\right] D_{r}^{+} \psi_{j}^{n}} \\
& \quad=i k_{0}\left[p_{0}-1-\left(p_{1}-q_{1}\right) V_{j}^{n+1 / 2}\right. \\
& \left.\quad+\left(p_{1}-q_{1}\right) k_{0}^{-2} \rho_{j} D_{z}^{0}\left(\rho_{j}^{-1} D_{z}^{0}\right)\right] \frac{\psi_{j}^{n}+\psi_{j}^{n+1}}{2}
\end{aligned}
$$

with $V_{j}^{n+1 / 2}:=V\left(z_{j}, r_{n+1 / 2}\right)$ and the difference operators

$$
\begin{aligned}
D_{r}^{+} \psi_{j}^{n} & =\frac{\psi_{j}^{n+1}-\psi_{j}^{n}}{\Delta r}, \\
D_{z}^{0} \psi_{j}^{n} & =\frac{\psi_{j+1 / 2}^{n}-\psi_{j-1 / 2}^{n}}{\Delta z}
\end{aligned}
$$

Proceeding similarly to the derivation of (4.12) one can show

$$
\begin{aligned}
D_{r}^{+} \sum_{j \in \mathbb{Z}} \frac{\left|\psi_{j}^{n}\right|^{2}}{\rho_{j}}= & -C_{1} k_{0}^{-1} \sum_{j \in \mathbb{Z}} \operatorname{Im}\left\{V_{j}^{n+1 / 2}\right\} \\
& \cdot\left|\psi_{j}^{n+1 / 2}+\frac{i q_{1}}{p_{1}-q_{1}} k_{0}^{-1} D_{r}^{+} \psi_{j}^{n}\right|^{2} \frac{1}{\rho_{j}}
\end{aligned}
$$

with $C_{1}=2\left(p_{1}-q_{1}\right)^{2} /\left(p_{1}-p_{0} q_{1}\right)$. Hence, the scheme (4.17) preserves the discrete weighted $L^{2}$-norm in the dissipation-free case ( $V$ real). 
Analogously to the discretization in [25] a discretized $T B C$ for the WAPE reads:

$$
\begin{aligned}
& \int_{0}^{r} \psi_{z}\left(z_{b}, r_{n}-\tau\right) \mathrm{e}^{i \theta \tau} \mathrm{e}^{i \beta \tau}\left[J_{0}(\beta \tau)+i J_{1}(\beta \tau)\right] d \tau \\
& \approx \sum_{m=0}^{n-1} \frac{\psi_{J}^{n-m}-\psi_{J-1}^{n-m}}{\Delta z} \\
& \quad \cdot\left[\tilde{J}_{0}\left(\beta r_{m+1 / 2}\right)+i \tilde{J}_{1}\left(\beta r_{m+1 / 2}\right)\right] \int_{r_{m}}^{r_{m+1}} \mathrm{e}^{i \theta \tau} d \tau,
\end{aligned}
$$

with the damped Bessel functions $\tilde{J}_{\nu}(z):=\mathrm{e}^{i z} J_{\nu}(z)$, $z \in \mathbb{C}$. This yields the following discretized TBC:

$$
\psi_{j}^{n}-\psi_{J-1}^{n}=\frac{i \Delta z}{\eta} \frac{\rho_{w}}{\rho_{b}} \psi_{j}^{n}-B^{\prime} \sum_{m=0}^{n-1}\left(\psi_{J}^{n-m}-\psi_{J-1}^{n-m}\right) \tilde{\ell}_{m},
$$

with

$$
\begin{aligned}
& B^{\prime}=i \beta \mathrm{e}^{(i / 2) \theta \Delta r} \frac{\sin (1 / 2 \theta \Delta r)}{(1 / 2) \theta} \\
& \tilde{\ell}_{m}=\mathrm{e}^{i \theta m \Delta r}\left[\tilde{J}_{0}\left(\beta r_{m+1 / 2}\right)+i \tilde{J}_{1}\left(\beta r_{m+1 / 2}\right)\right] .
\end{aligned}
$$

For calculating these convolution coefficients $\tilde{\ell}_{n}$ in far field simulations one has to evaluate $J_{\nu}(z)$ for large complex $z$ which is a rather delicate problem. Due to the exponential growth of the Bessel functions for fixed $\nu$ and $|z| \rightarrow \infty$ (see [1]) many standard software routines are not able to evaluate $J_{\nu}(z)$ for large complex $z$. For this reason we used a subroutine of Amos [3] to evaluate $\tilde{J}_{\nu}(z), \operatorname{Im} z \geq 0$.

The discrete exterior problem in the bottom region for $j \geq J$ :

$$
\left[R \delta_{b}+q \Delta^{2}\right]\left(\psi_{j}^{n+1}-\psi_{j}^{n}\right)=i\left[R \kappa_{b}+\Delta^{2}\right]\left(\psi_{j}^{n+1}+\psi_{j}^{n}\right),
$$

with

$$
\begin{gathered}
\delta_{b}=1-q_{1}\left(1-N_{b}^{2}\right), \quad R=\frac{2 k_{0}}{p_{1}-q_{1}} \frac{\Delta z^{2}}{\Delta r} \\
q=\frac{\Delta r}{2} \frac{q_{1}}{p_{1}-q_{1}} k_{0}^{-1} \\
\kappa_{b}=\frac{\Delta r}{2} k_{0}\left[p_{0}-1-\left(p_{1}-q_{1}\right)\left(1-N_{b}^{2}\right)\right] .
\end{gathered}
$$

The DTBC for the SPE and the WAPE then reads:

$$
(1+i q) \psi_{J-1}^{n}=\psi_{J}^{n} * \ell_{n}=\sum_{m=1}^{n} \psi_{J}^{m} \ell_{n-m}, \quad n \geq 1,
$$

with the convolution coefficients $\ell_{n}:=(1+i q)$ $\mathcal{Z}^{-1}\left\{\nu_{1}^{-1}(z)\right\}$ given by

$$
\begin{gathered}
\ell_{n}=\left[1+i q+\frac{i}{2}(\gamma-i \sigma) \mathrm{e}^{-i \xi}\right] \delta_{n}^{0}-\frac{i}{2} H(-1)^{n} \mathrm{e}^{i n \xi} \\
-\zeta\left\{Q_{n}(\mu)+\mathrm{e}^{-i \xi} \lambda^{-2} Q_{n-1}(\mu)+\omega \mathrm{e}^{-i \varphi}\right. \\
\left.\cdot \sum_{m=0}^{n-1}\left(-\mathrm{e}^{i \xi}\right)^{n-m} Q_{m}(\mu)\right\}
\end{gathered}
$$

$$
\begin{aligned}
& \gamma=R \delta_{b}, \quad \sigma=-R \kappa_{b}, \quad \lambda=\sqrt[+]{\frac{E}{G}} \\
& \mu=\frac{F}{\sqrt[+]{E G}}, \quad \omega=\frac{H^{2}}{|E|}, \\
& \xi=\arg \frac{1-i q}{1+i q}, \quad \varphi=\arg E \\
& \zeta=\frac{i}{2}|E|^{1 / 2} \mathrm{e}^{i \varphi / 2} \\
& E=(\gamma+i \sigma)[\gamma-4 q+i(\sigma+4)], \\
& F=\gamma(\gamma-4 q)+\sigma(\sigma+4), \\
& G=(\gamma-i \sigma)[\gamma-4 q-i(\sigma+4)], \\
& H=\gamma+i \sigma+(\gamma-i \sigma) \mathrm{e}^{i \xi} .
\end{aligned}
$$

In (4.23) $Q_{n}(\mu):=\lambda^{-n} P_{n}(\mu)$ denotes the damped Legendre polynomials $\left(Q_{0} \equiv 1, Q_{-1} \equiv 0\right)$. In the dissipative case $\alpha_{b}>0$ we have $|\lambda|>1, \mu$ becomes complex and $\left|P_{n}(\mu)\right|$ typically grows with $n$. In order to evaluate $\ell_{n}$ in a numerically stable fashion it is therefore necessary to use the damped polynomials $Q_{n}(\mu)$ in (4.23).

The convolution coefficients (4.23) behave asymptotically as

$$
\ell_{n} \cong-i H(-1)^{n} \mathrm{e}^{i n \xi}, \quad n \rightarrow \infty,
$$

which may lead to subtractive cancellation in (4.22) (note that $\psi_{J}^{m} \approx \psi_{J}^{m+1}$ in a reasonable discretization). Therefore we use the following 
numerically more stable fashion of the DTBC in the implementation:

$$
(1+i q) \psi_{J-1}^{n}-\ell_{0} \psi_{J}^{n}=(1-i q) \psi_{J-1}^{n-1}+\sum_{m=1}^{n-1} \psi_{J}^{m} s_{n-m},
$$

with $s_{n}:=\ell_{n}+\mathrm{e}^{\mathrm{i} \xi} \ell_{n-1}, n \geq 1$. The coefficients $s_{n}$ are calculated as

$$
\begin{aligned}
s_{n}= & {\left[(1+i q) \mathrm{e}^{i \xi}+\frac{i}{2}(\gamma-i \sigma)\right] \delta_{n}^{1} } \\
& +\zeta \frac{Q_{n}(\mu)-\lambda^{-2} Q_{n-2}(\mu)}{2 n-1} .
\end{aligned}
$$

Alternatively, they can be calculated directly with the recurrence formula

$$
s_{n}=\frac{2 n-3}{n} \mu \lambda^{-1} s_{n-1}-\frac{n-3}{n} \lambda^{-2} s_{n-2}, \quad n \geq 4,
$$

once $s_{1}, s_{2}, s_{3}$ are computed from (4.26). Using asymptotic properties of the Legendre polynomials one finds $s_{n}=O\left(n^{-3 / 2}\right), n \rightarrow \infty$ which agrees with the decay of the convolution kernel in the differential TBC (4.15).

Another problem is the discrete treatment of the (typical) density jump at the sea bottom $z=z_{b}$ which has to be incorporated into the DTBC (4.22). It is standard practice $[2,18]$ to use the usual grid $z_{j}, j \in \mathbb{N}_{0}$ and locate the discontinuity of $\rho$ at the grid point $z_{J}$ using $\rho_{J}=2 \rho_{b} \rho_{w} /\left(\rho_{b}+\rho_{w}\right)$. Here, as an alternative approach, we use an offset grid, i.e., $\tilde{z}_{j}=(j+1 / 2) \Delta z, \tilde{\psi}_{j}^{n} \sim \psi\left(\tilde{z}_{j}, r_{n}\right), j=-1(1) J$, where the water-bottom interface with the density jump lies between the grid points $j=J-1$ and $J$. For discretizing the matching conditions in this case one wants to find suitable approximations for $\psi$ and $\rho$ at the interface $z_{b}, \Psi \sim \psi\left(z_{b}\right)$ and $\rho_{\text {eff }}=$ $\rho\left(z_{b}\right)$, such that both sides of the discretized second matching condition (4.10b)

$$
\begin{aligned}
& \frac{1}{\rho_{w}} \frac{\tilde{\psi}_{J}^{n}-\Psi}{\Delta z / 2}=\frac{1}{\rho_{b}} \frac{\Psi-\tilde{\psi}_{J-1}^{n}}{\Delta z / 2} \text { are equal to } \\
& \quad \frac{1}{\rho_{\mathrm{eff}}} \frac{\tilde{\psi}_{J}^{n}-\tilde{\psi}_{J-1}^{n}}{\Delta z} .
\end{aligned}
$$

This approach results in an effective density $\rho_{\mathrm{eff}}=$ $\left(\rho_{w}+\rho_{b}\right) / 2$ (based on a different derivation this was also used in [10]). In numerical tests we found that the offset grid with the above choice of $\rho_{\text {eff }}$ produces slightly better results that have less Gibbs' oscillations at the discontinuity of $\psi_{z}$ at $z_{b}$. At the surface we use instead of $\psi_{0}^{n}=0$ the offset BC $\tilde{\psi}_{0}^{n}=-\tilde{\psi}_{-1}^{n}$.

Finally it remains to reformulate the DTBC (4.22) such that the density jump is taken into account. We rewrite the discretization of the second depth derivative at $j=J$ from (4.17):

$$
\begin{aligned}
(\Delta z)^{2} & {\left[\rho_{J} D_{z}^{0}\left(\rho_{J}^{-1} D_{z}^{0} \tilde{\psi}_{J}^{n}\right)\right] } \\
& =\Delta^{2} \tilde{\psi}_{J}^{n}+\left(1-\frac{\rho_{b}}{\rho_{\text {eff }}}\right)\left(\tilde{\psi}_{J}^{n}-\tilde{\psi}_{J-1}^{n}\right) .
\end{aligned}
$$

Comparing the r.h.s. of (4.29) to (4.21) we observe that only one additional term appears, and instead of (3.6) we get

$$
\begin{aligned}
& \hat{\tilde{\psi}}_{J+1}(z)-\left[1-i R \frac{\delta_{b}(z-1)-i \kappa_{b}(z+1)}{z+1+i q(z-1)}\right] \hat{\tilde{\psi}}_{J}(z) \\
& =\frac{\rho_{b}}{\rho_{\text {eff }}}\left(\hat{\tilde{\psi}}_{J}(z)-\hat{\tilde{\psi}}_{J-1}(z)\right) .
\end{aligned}
$$

Using $\hat{\tilde{\psi}}_{J+1}(z)=\nu_{1}(z) \hat{\tilde{\psi}}_{J}(z)$, where $\nu_{1}(z)$ denotes the solution of (3.7), and considering the fact that $\nu_{1}(z)+\nu_{1}^{-1}(z)$ is equal to the term in the squared brackets in (4.30) we obtain the $Z$-transformed DTBC:

$$
\begin{aligned}
\hat{\tilde{\psi}}_{J}(z)-\hat{\tilde{\psi}}_{J-1}(z)= & \frac{\rho_{\mathrm{eff}}}{\rho_{b}} \hat{\tilde{\psi}}_{J}(z) \\
& -\frac{\rho_{\mathrm{eff}}}{\rho_{b}} \nu_{1}^{-1}(z) \hat{\tilde{\psi}}_{J}(z) .
\end{aligned}
$$

Hence, the DTBC including the density jump reads

$$
\begin{aligned}
(1+i q) & \frac{\rho_{b}}{\rho_{\mathrm{eff}}} \tilde{\psi}_{J-1}^{n}+\left[(1+i q)\left(1-\frac{\rho_{b}}{\rho_{\mathrm{eff}}}\right)-\ell_{0}\right] \tilde{\psi}_{J}^{n} \\
= & -(1-i q) \frac{\rho_{b}}{\rho_{\mathrm{eff}}} \tilde{\psi}_{J-1}^{n-1} \\
& -(1-i q)\left(1-\frac{\rho_{b}}{\rho_{\mathrm{eff}}}\right) \tilde{\psi}_{J}^{n-1}+\sum_{m=1}^{n-1} \tilde{\psi}_{J}^{m} s_{n-m},
\end{aligned}
$$

with the convolution coefficients $s_{n}$ given by (4.26). 


\section{NUMERICAL EXAMPLES}

In the three examples of this Section we shall compare the numerical results from using our new discrete TBC to the solution using other discretization strategies of the TBC for the Schrödinger equation, the SPE and the WAPE.

Example 1 This example shows a simulation of a right travelling Gaussian beam $\left[\psi^{I}(x)=\exp \right.$ $\left.\left(i 100 x-30(x-0.5)^{2}\right)\right]$ at two consecutive times evolving under the free Schrödinger equation $(\hbar=1)$ with the rather coarse discretization $\Delta x=1 / 160, \Delta t=2 \cdot 10^{-5}$. Discretizing the analytic TBCs via (3.2) (scheme of Mayfield [17]) or as in Baskakov and Popov [7] induces strong numerical reflections. Our discrete TBCs (3.9), however, yield the smooth numerical solution to the whole-space problem, restricted to the computational interval $[0,1]$ (up to round-off errors).

Example 2 In the following two examples we used the Gaussian beam from [16] as initial data. Below we present the transmission loss $-10 \log _{10}|p|^{2}$, where the acoustic pressure $p$ is calculated from (4.3). Example 2 is a well-known benchmark problem from the literature $[16,21,25]$. In this example the ocean region $(0<z<240 \mathrm{~m})$ with the uniform density $\rho_{w}=1.0 \mathrm{gcm}^{-3}$ is modeled by the SPE (4.6). It contains no attenuation and a large density jump $\left(\rho_{b}=2.1 \mathrm{gcm}^{-3}\right)$ at the waterbottom interface. Hence, this problem provides a test of the treatment of the density jump in the TBCs applied along $z_{b}=240 \mathrm{~m}$.

The source of $f=100 \mathrm{~Hz}$ is located at a water depth $z_{s}=30 \mathrm{~m}$ and the receiver depth is at $z_{r}=$ $90 \mathrm{~m}$. The sound speed profile in water is given by $c(z)=1498+|120-z| / 60 \mathrm{~ms}^{-1}$, and the sound speed in the bottom is $c_{b}=1505 \mathrm{~ms}^{-1}$. For our calculations up to a maximum range of $20 \mathrm{~km}$ we used a reference sound speed $c_{0}=1500 \mathrm{~ms}^{-1}$ and a computational grid with depth step $\Delta z=2 \mathrm{~m}$ and range step $\Delta r=5 \mathrm{~m}$ (the same step sizes were used in [25]).

In Figure 2 the solid line is the solution with our new discrete TBC (4.32) and the dotted line is
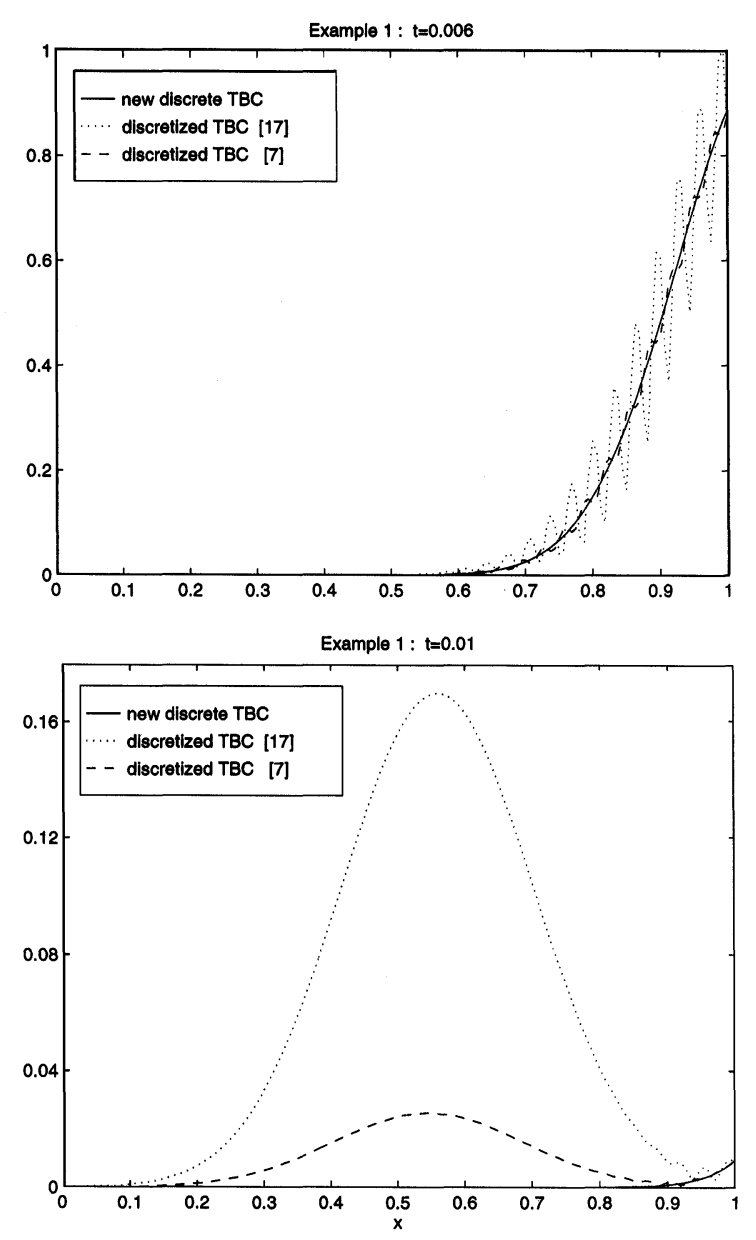

FIGURE 1 Solution $|\psi(x, t)|$ at time $t=0.006, t=0.1$ : the solution with the new discrete TBCs $(-)$ coincides with the whole-space solution, while the solution with the discretized analytic TBCs (3.2) from [17] ( $\cdots)$ or from [7] (---) introduces strong numerical reflections.

obtained with the discretized TBC (analogously to (3.3)). The discretized TBC clearly introduces a systematic phase-shift error, which is roughly proportional to $\Delta z$. The discretized TBC also produces artificial oscillations ( $c f$. the zoomed region), while our new DTBC yields the smooth solution with the same numerical effort.

Figure 3 shows the poor agreement of the solution using the discretized TBC with the computed half-space solution, which coincides with the solution using our new DTBC. 


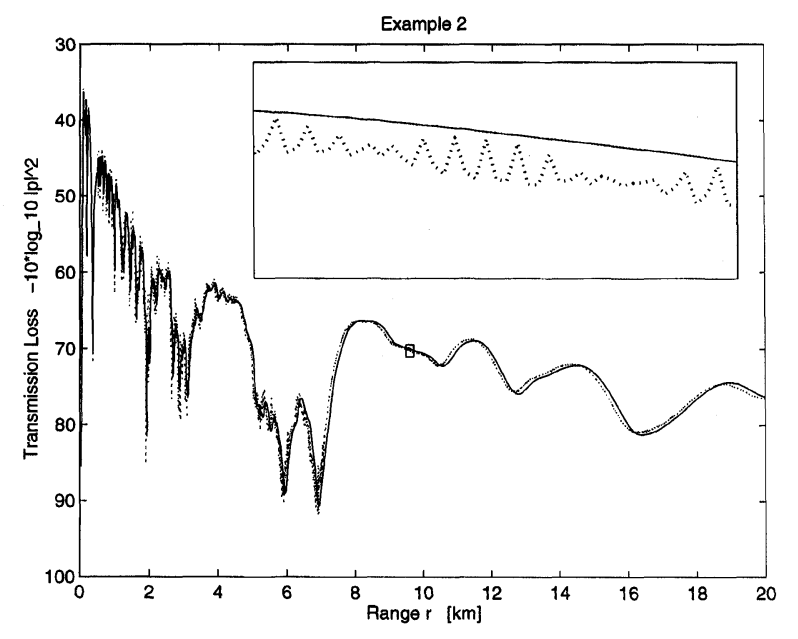

FIGURE 2 Transmission loss at $z_{r}=90 \mathrm{~m}$ for Example 2: the solution with the new discrete TBC (-) coincides with the halfspace solution, while the solution with the discretized TBC $(\cdots)$ introduces a phase-shift and artificial oscillations.

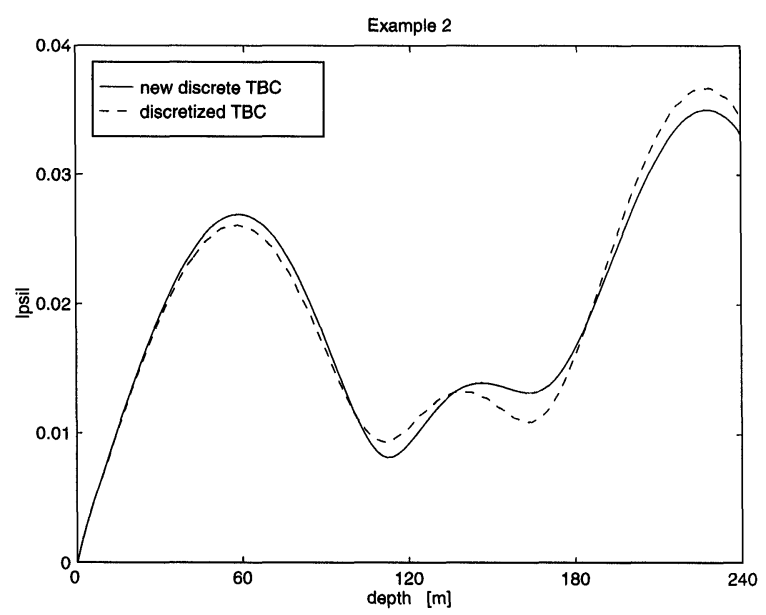

FIGURE 3 Vertical cut of the solution at $r=19 \mathrm{~km}$ for Example 2: $|\psi(z, r=19 \mathrm{~km})|$.

Example 3 This example appeared as the NORDA test case 3B in the PE Workshop I [16, 21, 25]. The environment for this example consists of an isovelocity water column $\left(c(z)=1500 \mathrm{~ms}^{-1}\right)$ over an isovelocity half-space bottom $\left(c_{b}=1590 \mathrm{~ms}^{-1}\right)$. The density changes at $z_{b}=100 \mathrm{~m}$ from $\rho_{w}=$ $1.0 \mathrm{gcm}^{-3}$ in the water to $\rho_{b}=1.2 \mathrm{gcm}^{-3}$ in the bottom. The source and the receiver are located at the same depth near the bottom: $z_{s}=z_{r}=99.5 \mathrm{~m}$.
The source frequency is $f=250 \mathrm{~Hz}$. The attenuation in the water is zero, and the bottom attenuation is $\alpha_{b}=0.5 \mathrm{~dB} / \lambda_{b}$, where $\lambda_{b}=c_{b} / f$ denotes the wavelength of sound in the bottom. Here, the steepest angle of propagation (which is the equivalent rayangle of the highest of the 11 propagating modes) is approximately $20^{\circ}(c f$. [25]). Since the source is located near the bottom, the higher modes are significantly excited. Therefore the wide angle capability is important here and we use the WAPE (4.8) (with the coefficients of Claerbout) to solve this benchmark problem.

The maximum range of interest is $10 \mathrm{~km}$ and the reference sound speed is chosen as $c_{0}=1500 \mathrm{~ms}^{-1}$. The calculations were carried out using $\Delta z=$ $0.25 \mathrm{~m}$ and $\Delta r=2.5 \mathrm{~m}$. Since the source is placed close to the bottom, the TBC was applied below the ocean-bottom interface (the same was done in [25]).

The typical feature of this problem is the large destructive interference null at a range of $7 \mathrm{~km}$. Figure 4 displays the transmission loss results from 5 to $10 \mathrm{~km}$.

Figure 5 shows the deviation of the solution with the discretized TBC compared to the

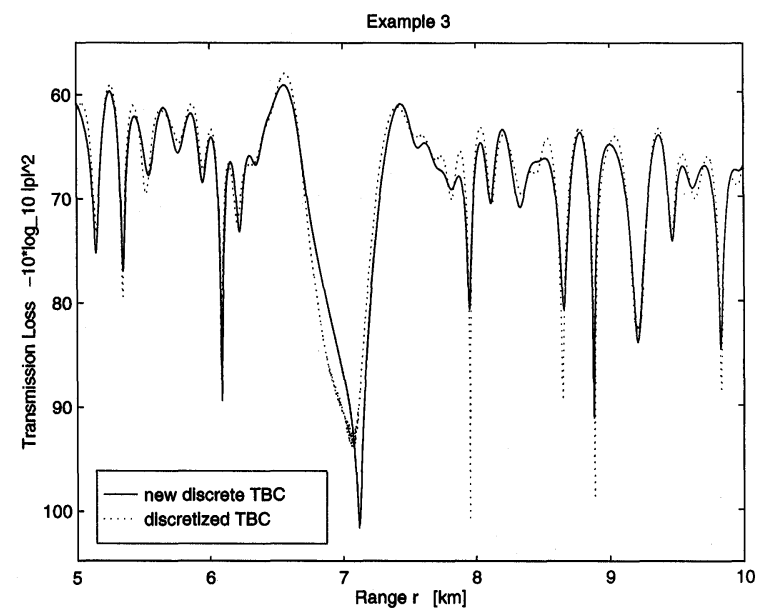

FIGURE 4 Transmission loss at $z_{r}=99.5 \mathrm{~m}$ for Example 3: the solution with the new discrete TBC coincides with the halfspace solution, while the solution with the discretized TBC still deviates significantly from it for the chosen discretization. 


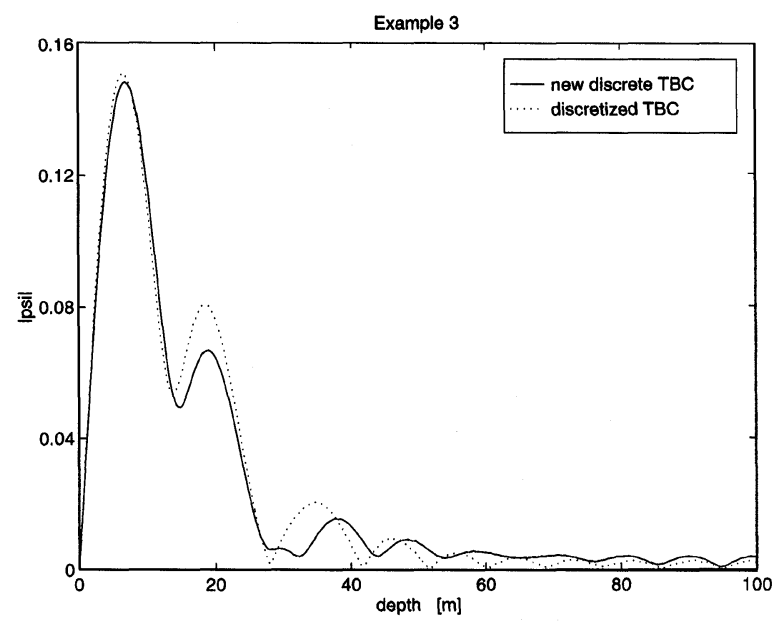

FIGURE 5 Vertical cut of the solution at $r=7 \mathrm{~km}$ for Example 3: $|\psi(z, r=7 \mathrm{~km})|$.

computed half-space solution, which coincides with the solution using our new discrete TBC.

\section{CONCLUSIONS}

We have derived a new discretization (discrete $T B C$ ) of the TBC for general Schrödinger-type equations. It is of discrete convolution form involving the boundary data from the whole past "history". The convolution coefficients $s_{n}$ are calculated via a simple three-term recurrence relation and they decay like $O\left(n^{-3 / 2}\right)$. Since our new DTBC has the same convolution structure as existing discretizations, it requires the same computational effort but improves two shortcomings: DTBCs are more accurate (in fact, as accurate as the discrete whole-space problem) and they yield an unconditionally stable scheme.

We point out that the superiority of DTBCs over other discretizations of TBCs is not restricted to (1.1) or to our particular interior discretization scheme (see e.g. $[4,11,12])$. Their applicability to other models only depends on the possibility to derive them explicitly; in our case the crucial point was to find the inverse $Z$-transformation of (3.8) explicitly. As a general philosophy, DTBCs should be used (if derivable) whenever highly accurate solutions are important.

\section{Acknowledgement}

The author thanks Dr. A. Arnold for many helpful suggestions to this article and acknowledges support by the DFG under Grant No. MA 1662/1-3.

\section{References}

[1] Abramowitz, M. and Stegun, I. A. (1965). Handbook of Mathematical Functions, National Bureau of Standards, Applied Math. Series, 55, Dover Publications.

[2] Akrivis, G. D., Dougalis, V. A. and Zouraris, G. E. (1996). Error Estimates for Finite Difference Methods for a Wideangle "Parabolic" Equation, SIAM J. Num. Anal., 33, $2488-2509$.

[3] Amos, D. E. (1986). Algorithm 644: A Portable Package for Bessel Functions of a Complex Argument and Nonnegative Order, ACM Trans. Math. Software, 12, 265-273.

[4] Arnold, A. (1998). Numerically Absorbing Boundary Conditions for Quantum Evolution Equations, VLSI Design, 6, 313-319.

[5] Arnold, A. and Ehrhardt, M. (1998). Discrete transparent boundary conditions for wide angle "parabolic" equations in underwater acoustics, J. Comp. Phys., 145, 611-638.

[6] Bamberger, A., Engquist, B., Halpern, L. and Joly, P. (1988). Parabolic wave equation approximations in heterogeneous media, SIAM J. Appl. Math., 48, 99-128.

[7] Baskakov, V. A. and Popov, A. V. (1991). Implementation of transparent boundaries for numerical solution of the Schrödinger equation, Wave Motion, 14, 123-128.

[8] Claerbout, J. F. (1970). Coarse grid calculation of waves in inhomogeneous media with application to delineation of complicated seismic structure, Geophysics, 35, 407-418.

[9] Claerbout, J. F. (1976). Fundamentals of Geophysical Data Processing, McGraw-Hill, New York.

[10] Collins, M. D. (1989). A higher-order parabolic equation for wave propagation in an ocean overlying an elastic bottom, J. Acoust. Soc. Am., 86, 1459-1464.

[11] Douglas, J. Jr., Santos, J. E., Sheen, D. and Bennethum, L. S. (1993). Frequency domain treatment of onedimensional scalar waves, Math. Mod. and Meth. in Appl. Sc., 3, 171-194.

[12] Ehrhardt, M. (1997). Discrete Transparent Boundary Conditions for Parabolic Equations, In: Proceedings of the GAMM 96 Conference, ZAMM, 77, Supplement 2, S543S544.

[13] Greene, R. R. (1984). The rational approximation to the acoustic wave equation with bottom interaction, $J$. Acoust. Soc. Am., 76, 1764-1773.

[14] Gripenberg, G., Londen, S. O. and Staffans, O. (1990). Voltera integral and functional equations, Cambridge Univ. Press, Cambridge. 
[15] Hellums, J. R. and Frensley, W. R. (1994). Non-Markovian open-system boundary conditions for the time-dependent Schrödinger equation, Phys. Rev. B, 49, 2904-2906.

[16] Lee, D. and McDaniel, S. T. (1987). Ocean acoustic propagation by finite difference methods, Comput. Math. Appl., 14, 305-423.

[17] Mayfield, B. (1989). Non-local boundary conditions for the Schrödinger equation, Ph.D. Thesis, University of Rhode Island, Providence, RI.

[18] McDaniel, S. T. and Lee, D. (1982). A finite-difference treatment of interface conditions for the parabolic wave equation: The horizontal interface, J. Acoust. Soc. Am., 71, 855-858.

[19] Papadakis, J. S. (1982). Impedance formulation of the bottom boundary condition for the parabolic equation model in underwater acoustics, NORDA Parabolic Equation Workshop, NORDA Tech. Note 143.

[20] Papadakis, J. S., Taroudakis, M. I., Papadakis, P. J. and Mayfield, B. (1992). A new method for a realistic treatment of the sea bottom in the parabolic approximation, J. Acoust. Soc. Am., 92, 2030-2038.

[21] Papadakis, J. S. (1992). Impedance Bottom Boundary Conditions for the Parabolic-Type Approximations in Underwater Acoustics, in Advances in Computer Methods for Partial Differential Equations VII, (Eds.) Vichnevetsky, R., Knight, D. and Richter, G., IMACS, New Brunswick, NJ, pp. 585-590.
[22] Schmidt, F. and Deuflhard, P. (1995). Discrete transparent boundary conditions for the numerical solution of Fresnel's equation, Comput. Math. Appl., 29, 53-76.

[23] Schmidt, F. and Yevick, D. (1997). Discrete transparent boundary conditions for Schrödinger-type equations, J. Comp. Phys., 134, 96-107.

[24] Tappert, F. D. (1977). The parabolic approximation method, in Wave Propagation and Underwater Acoustics, Lecture Notes in Physics 70, (Eds.) Keller, J. B. and Papadakis, J. S., Springer, New York, pp. 224-287.

[25] Thomson, D. J. and Mayfield, M. E. (1994). An exact Radiation Condition for Use with the A Posteriori PE Method, J. Comp. Acous., 2, 113-132.

[26] Vanbésien, O. and Lippens, D. (1995). Directional coupling in dual-branch electron-waveguide junctions, Phys. Rev. B, 52, 5144-5153.

\section{Author Biography}

Matthias Ehrhardt is a Ph.D. student at the Technical University of Berlin. His research centers on discrete artificial boundaries for evolution equations. 

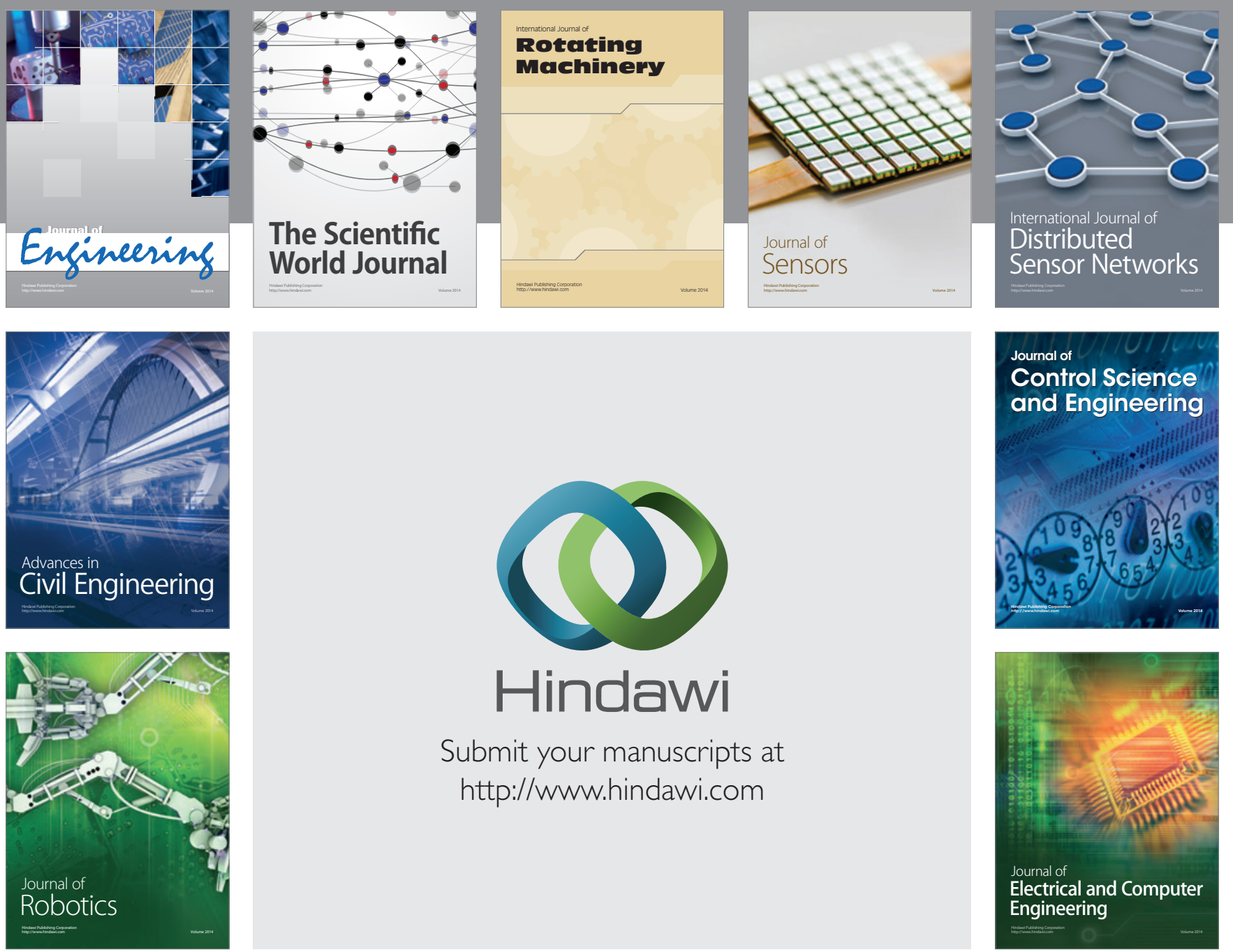

Submit your manuscripts at

http://www.hindawi.com
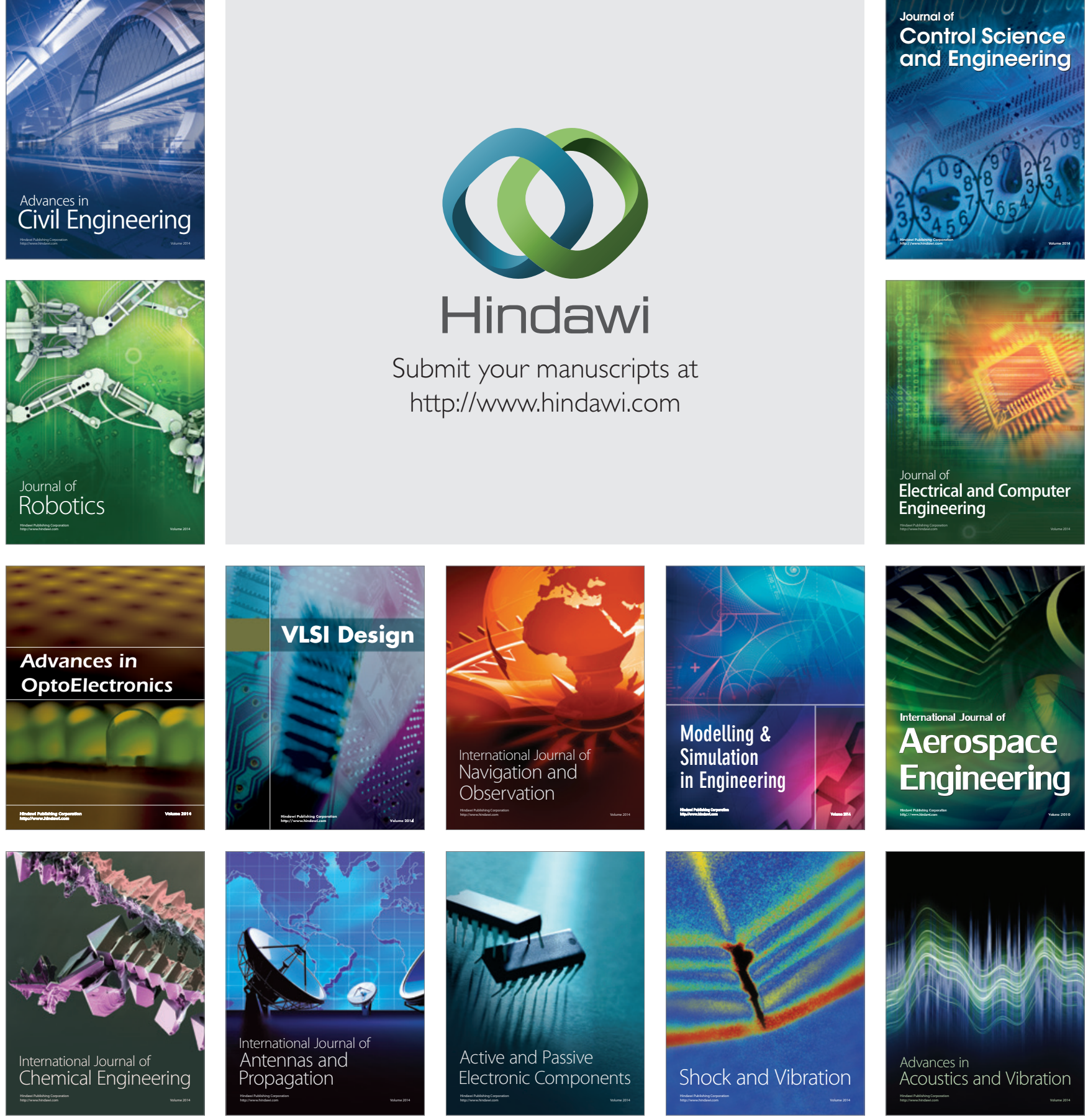